\title{
Forgetting faces over a week: Investigating self-reported face recognition ability and personality
}

\author{
Robin S S Kramer ${ }^{\text {Corresp. } 1}$ \\ ${ }^{1}$ School of Psychology, University of Lincoln, Lincoln, United Kingdom \\ Corresponding Author: Robin S S Kramer \\ Email address: remarknibor@gmail.com
}

Background. Although face recognition is now well studied, few researchers have considered the nature of forgetting over longer time periods. Here, I investigated how newly learned faces were recognised over the course of one week. In addition, I considered whether self-reported face recognition ability, as well as Big Five personality dimensions, were able to predict actual performance in a recognition task. Methods. In this experiment $(N=570)$, faces were learned through short video interviews, and these identities were later presented in a recognition test (using previously unseen images) after no delay, six hours, twelve hours, one day, or seven days. Results. The majority of forgetting took place within the first 24 hours, with no significant decrease after that timepoint. Further, self-reported face recognition abilities were moderately predictive of performance, while extraversion showed a small, negative association with performance. In both cases, these associations remained consistent across delay conditions.

Discussion. The current work begins to address important questions regarding face recognition using longitudinal, real-world time intervals, focussing on participant insight into their own abilities, and the process of forgetting more generally. 


\section{Forgetting faces over a week: Investigating self-}

\section{2 reported face recognition ability and personality}

3

4 Robin S. S. Kramer ${ }^{1}$

5

$6 \quad{ }^{1}$ School of Psychology, University of Lincoln, Lincoln, UK

7

8 Corresponding author:

9 Robin Kramer

10 School of Psychology, University of Lincoln, Brayford Pool, Lincoln, LN6 7TS, UK

11 Email address: remarknibor@gmail.com

12

13 


\section{Abstract}

15 Background. Although face recognition is now well studied, few researchers have considered 16 the nature of forgetting over longer time periods. Here, I investigated how newly learned faces

17 were recognised over the course of one week. In addition, I considered whether self-reported 18 face recognition ability, as well as Big Five personality dimensions, were able to predict actual performance in a recognition task.

Methods. In this experiment $(N=570)$, faces were learned through short video interviews, and these identities were later presented in a recognition test (using previously unseen images) after no delay, six hours, twelve hours, one day, or seven days.

Results. The majority of forgetting took place within the first 24 hours, with no significant decrease after that timepoint. Further, self-reported face recognition abilities were moderately predictive of performance, while extraversion showed a small, negative association with performance. In both cases, these associations remained consistent across delay conditions.

Discussion. The current work begins to address important questions regarding face recognition using longitudinal, real-world time intervals, focussing on participant insight into their own abilities, and the process of forgetting more generally.

\section{Introduction}

Although many researchers have argued that we are experts when it comes to perceiving and processing faces (e.g., Diamond \& Carey, 1986), more recent evidence suggests that this expertise may be limited to familiar faces only (Young \& Burton, 2018). Results have demonstrated that performance with familiar faces is significantly better in comparison with unfamiliar faces across a number of tasks, including recognition (Burton et al., 1999; Clutterbuck 
$37 \&$ Johnston, 2005; Ellis et al., 1979), sorting (Jenkins et al., 2011; Kramer et al., 2018), and

38 matching (Bruce et al., 2001; Ritchie et al., 2015).

39 Despite the important role that familiarity plays in face perception, surprisingly little is

40 known about the process of learning and familiarisation. Early studies emphasised the duration

41 or frequency of encounters (e.g., Shapiro \& Penrod, 1986), although this work had limited

42 success in providing a better understanding of the underlying mechanisms, perhaps due to their

43 reliance on highly standardised images. Recent research has shown that images of the same

44 person can appear very different (Jenkins et al., 2011), and that this within-person variability is

45 itself idiosyncratic (Burton et al., 2016). As such, increasing exposure to the different

46 appearances of a single face aids learning and subsequent recognition of that face (Andrews et

47 al., 2015; Ritchie \& Burton, 2017), presumably through the generation of a robust internal

48 representation (Burton et al., 2005). In contrast, limiting exposure to variability results in a

49 greater reliance on image-level properties (Hancock et al., 2000), causing difficulties when later

50 generalising to new instances.

51 While researchers are beginning to understand how learning and familiarisation can occur

52 over time and with exposure to a new face, few studies have considered the inverse process: how

53 faces are forgotten. Although several properties of the initial learning experience play an

54 important role (e.g., duration of exposure; for a review, see Deffenbacher et al., 2008), evidence

55 has also identified individual differences that influence forgetting, such as the level of stress felt

56 when a face is learned in an eyewitness context (Deffenbacher et al., 2004). It is likely that there

57 are also more stable differences across individuals that relate to the nature of face forgetting,

58 given the strong genetic (heritable) basis underlying face recognition ability (Shakeshaft \&

59 Plomin, 2015; Wilmer et al., 2010). 

employee's ability to recognise a face learned previously, it is important to determine whether

62 there are any easily measured predictors regarding performance. For example, individual

63 differences related to personality domains may be one such candidate (e.g., Lander \& Poyarekar,

64 2015), perhaps resulting from the above-mentioned genetic underpinnings of ability. Another 65 could be an individual's self-insight (e.g., Matsuyoshi \& Watanabe, 2020), making the selection

66 of workers far simpler if each was aware of his or her own abilities. However, as yet, no research

67 has considered how these factors may interact with the process of forgetting. Those who self68 report as demonstrating higher abilities with face recognition may be correct when faces were 69 learned only minutes ago, but such insights may be misplaced when targets have to be 70 remembered over the longer term. The same interaction could also be present for personality 71 domains, where specific traits are more strongly associated with face recognition (Lander \& 72 Poyarekar, 2015; Li et al., 2010), or memory performance (Stephan et al., 2020), but the 73 relationship between these three factors remains untested. The current experiment, therefore, will 74 investigate the process of face forgetting over longer time periods than are usually considered in 75 research designs, and will also begin to explore whether individual differences predict face 76 recognition ability over different retention intervals.

\section{Forgetting over time}

79 While numerous studies have focussed on face recognition, these have typically featured little or 80 no delay between learning and test (e.g., Baker et al., 2017; Duchaine \& Nakayama, 2006;

81 Lander \& Davies, 2007; Ritchie \& Burton, 2017; Rule et al., 2012; Russell et al., 2009; Zhou et 82 al., 2018). Of course, real-world recognition almost always involves some form of delay, which 
83 can often extend over many years. For this reason, Bahrick and colleagues (1975) used a cross-

84 sectional design in order to investigate retention intervals of up to 57 years by exploring

85 participants' recognition of yearbook photographs. Their findings suggested that under

86 conditions of prolonged acquisition (i.e., during participants' high school education), information

87 was preserved for much longer than laboratory demonstrations might show. Of course, the

88 insights gained through this type of design were at the expense of experimental control over

89 several variables.

90 A more recent study sought to balance the naturalistic learning and forgetting of faces over

91 several years with control over important factors that affect familiarity (Devue et al., 2019). The

92 researchers recruited participants who had watched all six seasons of the TV series Game of

93 Thrones, and subsequently tested their recognition across a variety of main and supporting

94 characters. Interestingly, although there were clear benefits due to increased and more recent

95 exposure, even well-learned faces were forgotten over time. In addition, the alteration of external

96 features (e.g., hair colour or accessories) led to a decrease in recognition for even the most

97 familiar faces, reiterating the findings mentioned earlier regarding the substantial effect of

98 within-person variability in appearance.

99 Despite the logistical difficulties involved with incorporating delays into experimental

100 designs, a number of studies have provided evidence of recognition after longer term intervals.

101 For example, Davis and Tamonytė (2017) asked participants to learn a target from a 1-min video

102 clip and subsequently identify the individual from a nine-person video line-up which took place

103 approximately ten days later. Accuracy on this task (in the 'no disguise' condition) was moderate

104 and depended on whether the target was present (33\%) or absent $(80 \%)$ in the line-up. Other

105 researchers have also employed longer delays across a variety of face recognition tasks (e.g., 28

Peer] reviewing PDF | (2021:02:57980:2:0:NEW 28 Jun 2021) 
106 days - Courtois \& Mueller, 1981; 23 days - Sauer et al., 2010; 35 days - Shepherd \& Ellis,

107 1973; 1 month - Shepherd et al., 1991; 4 months - Shepherd et al., 1982; 30 days - Yarmey,

108 1979), with a meta-analysis of these studies finding a small to medium association between

109 longer retention intervals and face forgetting (Deffenbacher et al., 2008).

110 Most relevant to the current work, Davis and colleagues (2020) constructed a face learning

111 task in order to investigate recognition after variable delay intervals. Each participant viewed ten

112 1-min video clips depicting the face and upper body of unfamiliar individuals, and were

113 subsequently tested on their recognition of these ten actors using six-person target-present video

114 line-ups. Importantly, the delay between learning and test varied from 1-182 days, representing a

115 significant period over which forgetting would occur. For the shortest delay (1-6 days), hits rates

116 were already low (0.46), decreasing even further $(0.26)$ for the longest delay group (56+ days).

117 In a second experiment, twenty unfamiliar individuals were learned using $30 \mathrm{~s}$ video clips, with

118 both target-present and target-absent photo line-ups presented at test. Retention intervals varied

119 from almost immediately to 50 days after learning. Even for those participants who were tested

120 within one day of learning, performance was poor (hits $=0.52$, correct rejections $=.32$ ), and for

121 those who were tested after 28 days or longer, performance had decreased even further (hits =

1220.27 , correct rejections $=.34$ ). It is clear from these results that the learning tasks were highly

123 difficult and showed increased forgetting over time, with retention intervals showing medium-

124 sized associations with hit rates in both experiments (Experiment 1: -0.31; Experiment 2: -0.43).

125 Although charting the progression of forgetting over longer periods of time is informative,

126 the majority of forgetting takes place during the first 24 hours (Deffenbacher et al., 2008). The

127 forgetting curve (Ebbinghaus, 1885) that has come to describe this deterioration over time is best

128 modelled by a power or exponential curve (Averell \& Heathcote, 2011; Rubin \& Wenzel, 1996; 
129 Wixted \& Ebbesen, 1991), with the steep early decline suggesting that this initial period should

130 be the focus of further exploration.

131

132 Self-report measures and face recognition ability

133 In recent years, researchers have increasingly focussed on whether people have accurate insights

134 into their face recognition abilities. Put simply, are participants aware of how well they perform

135 on such tasks? Although originally devised as a method of screening adults for developmental

136 prosopagnosia, scores on the 20-item prosopagnosia index (a measure of self-reported ability;

137 Shah, Gaule, et al., 2015) have since demonstrated medium-sized associations with performance

138 on the Glasgow Face Matching Test (Burton et al., 2010) and the Cambridge Face Memory Test

139 (CFMT; Duchaine \& Nakayama, 2006) in the general population (Gray et al., 2017; Livingston

140 \& Shah, 2018; Shah, Sowden, et al., 2015; Ventura et al., 2018). These results suggest that

141 participants do indeed have some level of meta-cognitive insight into their own abilities.

142 Similarly, scores on a 15-item questionnaire developed in a Hong Kong population for the

143 screening of congenital prosopagnosia (HK15; Kennerknecht et al., 2008) have since shown

144 small associations with the CFMT in typical adults (Palermo et al., 2017). Interestingly, after

145 removal of four dummy questions that were irrelevant with respect to face recognition, the

146 resulting 11-item subset of questions (HK11) showed a medium-sized association with an East

147 Asian version of the CFMT (Matsuyoshi \& Watanabe, 2020). Although these questionnaires are

148 those most frequently utilised as a measure of insight, other tools have also been developed with

149 some success (Arizpe et al., 2019; Bobak et al., 2019; Saraiva et al., 2019). Taken together, it

150 seems clear that, to some extent, people are aware of their face recognition abilities. 
152 of face recognition without delays (e.g., the CFMT). There is no evidence that self-reported

153 abilities show an association with performance when the task involves learning and then later

154 recognition following a delay. In a recent pre-print (Davis et al., 2019), researchers included a

155 single-item measure of insight in order to determine whether participants believed they were

156 below or above average in face recognition ability (using a 1-5 scale) and investigated the

157 recognition of learned identities up to six months later (see also Davis et al., 2020). However,

158 this measure of insight failed to predict accuracy on the task. Of course, this may be due to the

159 use of a single item for quantifying insight, and the question remains as to whether better

160 established measures are able to predict performance in this domain.

161 Related, there is some evidence that self-report measures of personality may also be 162 associated with performance on face-related tasks, although results appear to be mixed. For 163 example, extraversion may be related to face recognition (Lander \& Poyarekar, 2015; Li et al., 164 2010), while face matching does not appear to be related to personality measures, other than

165 perhaps facets of neuroticism (anxiety - Megreya \& Bindemann, 2013; no associations - Lander 166 \& Poyarekar, 2015). More recently, no relationship was found between personality factors and 167 measures of face memory and matching (McCaffery et al., 2018). When searching for faces in 168 crowds, there is also evidence that personality may (Kramer et al., 2020) or may not (Davis et al., 169 2018) be associated with performance measures. Again, there is a lack of research investigating 170 whether personality facets are related to performance on a recognition task over a time delay. 171

\section{The current experiment}


173 In light of the unanswered questions highlighted above, I identified two aims for the current

174 experiment, which can be summarised as follows. First, little is known regarding the nature of

175 face forgetting over the first 24 hours post-learning. As such, this study focussed on the first

176 week following exposure to these new identities, but with the specific goal of understanding this

177 crucial, initial time period. In order to learn more about real-world forgetting, participants

178 learned faces from short video interviews, comparable with exposure during a brief conversation.

179 Second, although several studies have now found evidence that self-reported face recognition

180 abilities, and personality measures to a lesser extent, were moderately predictive of actual

181 performance, the tasks employed in those experiments did not include any form of delay between

182 learning and test. As such, it remains unclear as to whether insight into one's own ability and

183 personality are predictive when incorporated into a more ecologically valid design, and whether

184 any such associations are altered by the retention interval.

186 Method

187 Participants

188 After restricting eligibility to those located in the USA, the UK, Australia, Canada, and New 189 Zealand (unless otherwise specified - see below), where the majority of residents speak English, 190 2,085 participants were recruited through Amazon Mechanical Turk (MTurk). Of these, 570 (231 191 women; age $M=39.2$ years, $S D=11.8$ years; $78.4 \%$ self-reported ethnicity as White) completed 192 the full study (both learning and test sessions), correctly answered all attention checks, and were 193 unfamiliar with all of the identities used as stimuli. Table 1 provides a summary for each 194 condition in terms of sample sizes, attrition rates, and exclusions. Participants who completed the 195 learning session were paid US $\$ 0.75$, and those who completed the test session received a further 
196 US $\$ 0.75$. Combined, this wage equated to approximately $\$ 6$ per hour, although the second test

197 session took less time but was priced equally in order to encourage participants to complete both

198 sessions. No payment was given for a session in which attention checks were answered

199 incorrectly. Participants provided informed consent online prior to taking part, and received an

200 online debriefing upon completion, in line with the university's ethics protocol. Ethical approval

201 for this experiment was granted by the University of Lincoln's ethics committee (ID 3508).

202 MTurk is a platform well-suited to longitudinal research (Chandler \& Shapiro, 2016;

203 Cunningham et al., 2017), and retention rates here (see Table 1), as with other studies (Shapiro et 204 al., 2013; Stoycheff, 2016), were relatively high. In order to maximise the likelihood that those 205 who participated in the learning session would return to complete the test session, MTurk 206 workers who had completed the first session were contacted individually using the 'pyMTurkR' 207 package (Burleigh \& Leeper, 2020) and invited to complete the second session.

208 An a priori power analysis was conducted using G*Power 3.1 (Faul, Erdfelder, Lang, \& 209 Buchner, 2007), based on the correlation between self-reported face recognition abilities (using 210 the same questionnaire featured here) and actual performance in previous work (-.38 -

211 Matsuyoshi \& Watanabe, 2020). In order to achieve $80 \%$ power at an alpha of .05, a total sample

212 size of 49 was required. As such, I aimed to recruit a minimum of 49 participants (after

213 exclusions) in each delay condition (described below).

214

215 Materials

216 From a larger database of 80 White (non-UK) European individuals (predominantly German or

217 Dutch), ten identities (four women) were chosen to serve as faces to be learned in the current

218 experiment. This selection was based upon the performance of a different sample of participants, 
219 where these identities were chosen to represent a range of face memorability scores, although

220 this was not investigated here. All were identified as nationally well-known (e.g., singers, actors,

221 athletes) while none had reached international levels of fame. For each person, a video interview

222 was found on YouTube in which they were filmed for at least one minute using a fixed camera

223 (i.e., the viewing angle of their face remained unchanged throughout) and spoke in a language

224 other than English (simulating natural conversation without the audio content providing

225 additional information that might aid learning). In all cases, the interviewer was positioned close

226 to the camera, resulting in a view of the face that was relatively front-on.

227 For each identity, a continuous $30 \mathrm{~s}$ segment was selected from the initial YouTube video

228 in which the person was predominantly front-on and speaking for most or all of the time (rather

229 than simply listening to the interviewer). The video was also cropped to $350 \times 350$ pixels in order

230 to include only the head and the top of the shoulders (and the background contained within that

231 frame; see Figure 1). These videos were in colour and included the audio information.

232 In order to create the recognition test, 20 additional identities (11 women) from the original

233 database were chosen at random with the caveat that half of the final set of 30 identities were

234 women. For each of these 30 people, a high-quality, colour photograph was downloaded from

235 Google Images in which they were approximately forward-facing. For the ten identities to be

236 learned, these photographs were chosen so that their appearance resembled that of the videos in

237 which they featured, e.g., matched for age, hair style, facial hair and glasses where applicable,

238 etc. Importantly, these were images taken in new contexts in all cases, and were not still frames

239 from the videos. Images were subsequently cropped to 350 x 350 pixels, displaying only the

240 head and the top of the shoulders (and the background contained within that frame; see Figure 1). 
242 questionnaire (e.g., "it takes me a long time to recognise people”; Kennerknecht et al., 2008). For

243 each item, participants select a response from the following: strongly agree, agree, uncertain,

244 disagree, strongly disagree. After reverse coding eight items, overall score is calculated by

245 summing individual responses, with lower scores indicating higher self-reported estimates of

246 face recognition ability. Subsequent removal of four dummy questions that are irrelevant with

247 respect to face identity recognition (e.g., "I get lost in new places") produces an 11-item subset

248 of questions (HK11; score range 11-55) which has previously shown a medium-sized association

249 with actual face recognition performance $(r=-0.38$; Matsuyoshi \& Watanabe, 2020). The HK11

250 demonstrates high levels of reliability (Cronbach's $\alpha=0.84$; Matsuyoshi \& Watanabe, 2020).

251 In order to measure participants' self-reported Big Five personality domains, I used the

252 Ten-Item Personality Inventory (TIPI; Gosling et al., 2003). For each item, participants respond

253 using a 1 (disagree strongly) to 7 (agree strongly) Likert scale. Participants are instructed to rate

254 the extent to which pairs of traits apply to them, e.g., "I see myself as extraverted, enthusiastic."

255 After reverse coding five items, the overall score on each domain is calculated by averaging the

256 two individual responses, with higher scores indicating higher self-perceived applicability for

257 that domain. This questionnaire is a short measure when compared with most personality

258 inventories, but strong correlations have been shown between the TIPI dimensions and the well-

259 validated 60-item NEO-PI-R (Costa \& McCrae, 2008; Erhart et al., 2009), as well as the 40-item

260 EPQ-R (Eysenck \& Eysenck, 1993; Holmes, 2010). With only two items per scale, the TIPI

261 demonstrates low reliability (Gosling et al., 2003), with Cronbach's $\alpha$ values of 0.68

262 (extraversion), 0.40 (agreeableness), 0.50 (conscientiousness), 0.73 (emotional stability), and

2630.45 (openness to experience). However, scales with small numbers of items commonly show 
264 low alpha scores (Gosling et al., 2003) and so test-retest reliability ( $r=.72$ over a six-week span)

265 is considered a more appropriate measure of an instrument's quality. Therefore, while providing

266 a similar measure to longer inventories, the benefit of its use here is its minimal demands on

267 participant time, requiring approximately one minute to complete.

268

269 Procedure

270 The experiment was completed using the Gorilla online testing platform (Anwyl-Irvine et al.,

271 2020). I collected information regarding the participant's age, gender, and ethnicity, as well as

272 their MTurk Worker ID. By assigning a 'qualification' using this Worker ID, I was able to

273 associate data files across learning and test sessions, as well as prevent participants from taking

274 part in more than one delay condition. These conditions comprised no delay, six hours, twelve

275 hours, one day, and seven days, with assignment to condition described below.

276 Participants first completed the TIPI and HK15 questionnaires in order that their

277 experience with the recognition task did not affect their self-estimates of ability. Next, they were

278 shown the ten $30 \mathrm{~s}$ videos in a random order and instructed, "Please watch the videos carefully

279 and learn to recognise each person's face." Participants were also asked to view the videos with

280 the sound enabled in order to make the learning experience more natural, although they were not

281 expected to understand what was being said (given that the spoken language was not English). A

282 'play video' button took participants to a new screen where the video started playback for each

283 learning trial, allowing participants to control their progress. However, once started, videos could 284 not be paused, rewound, or replayed.

285 Two attention checks were included during learning, appearing before the fourth and

286 eighth video presentations, given that attentiveness is a common concern when collecting data 
287 online (Hauser \& Schwarz, 2016). Each of these two trials instructed the participant to click on 288 either the 'left' or 'right' button presented onscreen. For instance, "Attention Check: Please click 289 the LEFT button now (in less than 10 seconds) to show you're paying attention" was displayed 290 onscreen. By requiring participants to respond within this limited time window, I could identify 291 those who were not paying attention or may have started videos and then pursued other activities. 292 For participants in the 'no delay' condition, the learning task was immediately followed by 293 the recognition test. Participants were presented with the 30 test images and asked to decide 294 whether the face was seen during learning or not. Responses were provided using a labelled 295 rating scale: 1) I'm sure it's someone I learned; 2) I think it's someone I learned; 3) I don't 296 know; 4) I think it's someone I didn't learn; 5) I'm sure it's someone I didn't learn.

297 Two additional trials were also included as attention checks during the recognition test.

298 Each of these two trials consisted of a celebrity's photograph (not one of the original 30), similar 299 in appearance to a real trial (background present, identical image size). However, the internal 300 features of the face were replaced with text, instructing the participant to respond with either ' 2 ' 301 or '4' on the response scale. For instance, "Attention Check: Please respond with '2' here." By 302 requiring participants to give different responses across the two attention checks, I could identify 303 those who were not paying attention or clicked the same button onscreen throughout the 304 experiment irrespective of what was being displayed. The presentation order of the 32 images was randomised for each participant. Responses were given using the mouse and were self-paced. Finally, participants were asked how many (if 307 any) of the faces they had recognised from their experiences prior to the experiment. For participants in conditions with a delay between the learning and test sessions, the

309 familiarity question directly followed learning (with no test included). During the separate test 
310 session, participants completed demographic information again (but did not repeat the two

311 questionnaire measures), followed by the recognition test and then another familiarity question.

312 Regarding knowledge about a subsequent test, all participants were informed onscreen at

313 the start of the learning task that there would be a recognition test afterwards. However, for those

314 in conditions with a delay, participants were simply told that this test would take place "in the

315 next several weeks" and were therefore unaware of the specific length of delay to which they

316 were assigned. For logistical reasons (e.g., making the experiment available for certain periods of

317 the day, keeping track of completions and inviting the appropriate participants to the test session,

318 etc.), rather than randomly allocating participants to conditions, recruitment for the five delay

319 conditions took place in the following sequence: no delay, one day, seven days, six hours, twelve 320 hours.

321 For the 'one day' and 'seven days' conditions, both the learning and test sessions were

322 each made available for approximately 24 hours or until no additional MTurk workers had taken

323 part for approximately two hours. In all conditions, if a sufficient sample size had not been

324 reached then the process of recruitment for both sessions was repeated as necessary. As

325 mentioned above, for the test sessions, qualifying participants were notified via email as the

326 session was posted on MTurk. However, for the 'six hours' and 'twelve hours' delays, both the

327 learning and test sessions were made available for only 1.5 hours each. Again, qualifying

328 participants were notified as the test session was made available and, for these conditions, were

329 informed that it would only be accessible for the next hour and a half. In order to avoid

330 participants completing the learning session who would not be available to complete the test

331 session six or twelve hours later due to the time of day (i.e., requiring one session to take place

332 during the night), MTurk workers from Australia and New Zealand were excluded from 
333 recruitment for these two conditions only (given the difference in time zones between these two

334 countries and the other three). In addition, for the 'twelve hours' condition, session timings were

335 chosen in order to avoid including a night's sleep.

336 Participants were prevented from completing the experiment using mobile phones (via

337 settings available in Gorilla) in order to ensure that videos and images were viewed at an

338 acceptable size onscreen.

339

340 Results

341 Data analysis included only those participants who correctly answered all attention checks and

342 reported being unfamiliar with all of the identities used as stimuli (see Table 1).

343 For each participant, I calculated the hit and false alarm rates for each possible threshold

344 (i.e., the theoretical boundary between 'learned' and 'new') along the recognition response scale

345 (1 through 5). Rather than making explicit judgements about whether identities were learned or

346 new, participants rated the likelihood that each identity had been learned. This approach,

347 therefore, focussed on their internal representation of this likelihood (a continuous measure)

348 rather than forcing a binary decision based on an internal threshold that differentiates a 'learned'

349 from a 'new' identity. Plotting these values produced the receiver operating characteristic

350 (ROC), with the area under this ROC curve (AUC) representing a measure that is widely used to

351 assess the performance of classification rules over the entire range of possible thresholds

352 (Krzanowski \& Hand, 2009). As such, AUC allowed quantification of the performance of a

353 classifier (here, each participant), irrespective of where the cut-off between binary

354 'learned'/'new' responses might have been placed. This more fine-grained analysis bypassed the

355 need to rely on a participant's final decision ('learned'/'new') in favour of investigating what 
356 was presumably the underlying perception - the likelihood that this identity was someone

357 previously learned. These data are summarised in Table 2, along with descriptive statistics for

358 the questionnaire responses. As Table 2 illustrates, the current sample scored lower on both

359 extraversion and openness in comparison with population norms (extraversion $=4.44$, openness

360 =5.38; Gosling et al., 2003), while HK11 scores were similar to those reported in the original

361 study $(M=24.04$; Matsuyoshi \& Watanabe, 2020).

362 In order to investigate whether performance (AUC) differed across the five delay

363 conditions, I carried out a univariate analysis of variance (ANOVA), which showed a statistically

364 significant main effect of delay, $F(4,565)=23.60, p<.001, \eta^{2}=0.14$. Pairwise comparisons

365 (Bonferroni corrected) revealed that the shortest conditions (no delay, 6 hours, 12 hours) all

366 differed from the longest conditions (1 day, 7 days; all $p \mathrm{~s}<.001)$. However, I found no

367 differences within these two subcategories (shortest delays: all $p \mathrm{~s}>.086$; longest delays: $p=$

368 1.00). The five conditions are shown in Figure 2.

369 Next, I considered whether self-reported face recognition ability (HK11) and Big Five

370 personality domains were associated with performance (AUC) across the whole sample.

371 Correlations with AUC were as follows: HK11, $r(568)=-.34, p<.001$; extraversion, $r(568)=-$

$372.12, p=.004$; agreeableness, $r(568)=.17, p<.001$; conscientiousness, $r(568)=.17, p<.001$;

373 emotional stability, $r(568)=.09, p=.027$; and openness, $r(568)=.12, p=.006$. However, after

374 applying Bonferroni correction for multiple tests, the correlation with emotional stability was no

375 longer statistically significant.

376 I then investigated whether self-reported face recognition ability and personality domains

377 predicted performance after controlling for differences as a result of delay condition. To this end,

378 I carried out a hierarchical linear regression, including delay condition (reference category: no 
379 delay) as the initial predictor (replicating the above ANOVA). For each of the six additional

380 predictors (HK11 scores and the five personality domain scores), I compared the model in which

381 it was included to the previous model in which it was absent (using the anova function in R). If

382 the model's improvement was statistically significant, this process was repeated in order to

383 consider the inclusion of the predictor's interaction with delay condition. This process, along

384 with the final model, $F(6,563)=35.29, p<.001, R^{2}=0.27$, can be seen in Table 3 , where only

385 HK11 and extraversion were included (Step 3). All other predictors (agreeableness,

386 conscientiousness, emotional stability, openness) and their interactions with delay failed to

387 significantly improve the model (all $p \mathrm{~s}>.05$ ). As such, the relationships between HK11 and

388 AUC, as well as extraversion and AUC, were shown to be consistent across delay conditions. As

389 Table 3 illustrates, HK11 scores were a stronger predictor of performance in comparison with

390 extraversion. In addition, although the inclusion of extraversion significantly improved the fit of

391 the model, the increase in $R^{2}(0.02)$ was small.

392 Finally, Figure 2 illustrates the forgetting curve (Ebbinghaus, 1885) for these data,

393 generated using MATLAB's fit function (model fit, $R^{2}=0.81$ ). The model includes a vertical

394 shift, as well as a horizontal shift in order to allow for a delay of zero (Averell \& Heathcote,

395 2011; Wixted \& Ebbesen, 1991). Power functions are generally accepted as suitable models for

396 forgetting, in particular when averaging across participants and therefore focussing on group-

397 level performance (Averell \& Heathcote, 2011; Murre \& Chessa, 2011; Wixted \& Ebbesen, 398 1991).

399

400 Discussion 
401 The experiment presented here was designed with two main aims. First, I investigated whether

402 self-report measures were predictive of face recognition abilities, even when learning and test

403 were separated by a delay. Second, I was interested in mapping the general process of forgetting

404 over time, with particular focus on the first 24 hours after initial exposure.

405 Recent research has found a medium-sized association between self-reported ability (using

406 the HK11 questionnaire) and actual face recognition performance (Matsuyoshi \& Watanabe,

407 2020). Indeed, several researchers have demonstrated similar-sized correlations between various

408 self-report instruments and different measures of performance (Arizpe et al., 2019; Bobak et al.,

409 2019; Gray et al., 2017; Livingston \& Shah, 2018). Here, I demonstrated that this association

410 remained when delay intervals were introduced. Indeed, the relationship between participants'

411 self-reported abilities and their actual performance was constant across these different delays.

412 This is an important result since the only previous study to consider this issue (Davis et al., 2019)

413 found no evidence of insight after delays, although the authors acknowledged that this may have

414 been due to the use of a single item to quantify insight.

415 In the current work, I found that HK11 scores provided a measure of self-reported ability

416 that was moderately predictive of performance, no matter whether recognition was required

417 immediately or after a delay of up to seven days. That the relationship remained constant across

418 the different delays is both novel and interesting, given that previous research has not considered

419 the possibility that the accuracy of self-report measures may be dependent on the interval

420 between learning and test. Clearly, memory requirements varied across the delay intervals used,

421 and these findings suggest that participants may incorporate this notion of recognising faces over

422 unspecified amounts of time into their responses. Indeed, HK11 items do not refer to specific

423 time delays and so it might be interesting to consider whether self-report measures that do 
424 specify the interval under consideration (i.e., asking only about recognition abilities a week after

425 meeting someone) could lead to more accurate insights regarding the specific delay in question,

426 although this remains to be investigated.

427 Regarding real-world applications, it is important that measures of insight extend beyond

428 tests of immediate recognition since screening individuals for their abilities, for example, would

429 almost certainly be with the intention of employing their skills in contexts involving substantial

430 delays (Davis et al., 2016). It is likely that the requirements involved in learning a face, and then

431 having to recognise that face immediately afterwards, are very different from those whereby

432 targets are recognised weeks or months later, as were the conditions faced by police officers

433 investigating the 2011 London riots (Davis, 2019), for example.

434 In addition to self-reported abilities, there is some evidence to suggest that particular facets

435 of personality may be associated with those who perform better on face-related tasks.

436 Extraversion may be one such candidate, showing associations with abilities in both face

437 recognition (Lander \& Poyarekar, 2015; Li et al., 2010) and spotting faces in crowds (Kramer et

438 al., 2020), although such evidence is far from conclusive (Davis et al., 2018; McCaffery et al.,

439 2018). Here, I found a small association between this dimension and recognition performance.

440 However, while the association remained constant across the five delay conditions, it was

441 somewhat surprising that extraversion showed a negative correlation with performance (although

442 see Kramer et al., 2020). Clearly, there is a need to investigate this result further since it seems

443 counterintuitive that introverted people may perform better with learning and later recognising

444 faces. One explanation is that extraversion itself may comprise two subcomponents (Bornstein et

445 al., 2011; Roberts et al., 2006): social dominance (surgency, assertiveness) and social vitality

446 (sociability, fun-seeking). For this reason, an overall measure of this dimension could be difficult 
447 to interpret and might mask different underlying associations with recognition ability. An

448 additional issue to note is that, as a subsample, MTurk workers are typically more introverted

449 than the general population (Burnham et al., 2018), which could limit the conclusions drawn

450 from experiments recruiting from this particular participant pool. Indeed, Table 2 suggests that

451 the current sample scored lower on both extraversion and openness in comparison with

452 population norms (Gosling et al., 2003). Therefore, although personality does appear to predict

453 face recognition ability in this experiment, I recommend further research in order to address this

454 issue more conclusively.

455 This experiment also aimed to explore the forgetting of faces longitudinally after realistic

456 learning. To this end, I utilised short video interviews where individuals were speaking and

457 facing towards the camera in order to simulate a brief real-world encounter. Previous research

458 has shown that the process of forgetting typically follows a power or exponential curve (e.g.,

459 Averell \& Heathcote, 2011; Rubin \& Wenzel, 1996; Wixted \& Ebbesen, 1991), with a steep

460 early decline. However, no research to date has explicitly investigated the forgetting curve

461 associated with faces and its formulation. While Deffenbacher and colleagues (2008) attempted

462 to model forgetting functions using several datasets, the authors were forced to estimate

463 performance after 'no delay' due to a lack of data and fitted their functions "by eye” (p. 145).

464 Importantly, these earlier experiments involved learning faces under conditions that failed to

465 mirror the real world (e.g., through the use of the same static images at learning and test). In line

466 with general predictions regarding forgetting, my results revealed that performance fell

467 dramatically within the first 24 hours. In addition, the deterioration between those tested in the

468 first 12 hours and those tested after 24 hours was also significant. After this point, no further

469 deterioration was seen during the subsequent 1-7 day period. 
An interesting issue to consider, although beyond the remit of the current work, is the

471 function of sleep for those who participated in the ' 1 day' and ' 7 days' conditions. (The timings

472 of the ' 12 hours' condition were chosen to avoid including a night's sleep for participants, who

473 were restricted to the USA, the UK, and Canada.) While previous work has suggested that face

474 learning may benefit from memory consolidation during sleep (Wagner et al., 2007), more recent

475 research has argued that, instead, it is wakefulness during retention that diminishes memory for

476 faces (Sheth et al., 2009). Ongoing sensory stimulation interferes with visual memory while

477 sleep shelters the individual from this interference. Although the current findings are in line with

478 previous research on sleep and wakefulness effects, further work might incorporate this factor in

479 order to investigate face forgetting during this first 24-hour period, e.g., by equating retention

480 intervals while manipulating the presence/absence of sleep.

481 In order to simulate a brief real-world encounter while minimising the influence of the

482 content of the conversation on learning/remembering, videos were constructed in which

483 identities spoke in languages other than English (predominantly German or Dutch), while

484 participants were recruited from countries where English is the primary language (the USA, the

485 UK, Australia, Canada, and New Zealand). However, it is possible that a small number of

486 participants understood what one or more of the targets were saying, and conversely, it may be

487 that some participants did not have the sound enabled during the task (although they were

488 instructed to do so). While previous research has demonstrated the beneficial role of motion in

489 learning new faces (Lander \& Bruce, 2003; Lander \& Davies, 2007), to my knowledge, there is

490 no research investigating whether the presence of speech aids face learning. In the current

491 experiment, enabling sound during learning may simply have better captured participants'

492 attention, although it is possible that an additional understanding of what the identities were 
493 saying (while likely infrequent due to recruitment restrictions) could have helped with learning 494 those particular faces. Even so, future research may consider whether the inclusion of additional 495 information learned through speech could benefit learning and later recognition.

496 Regarding the time taken to forget, it is worth noting that the identities used here were 497 national celebrities, chosen for logistical reasons - the availability of both naturalistic images and 498 video interviews. As such, it may be the case that these people were not representative of the 499 general population in terms of attractiveness and/or distinctiveness, both of which are known to 500 affect face memory (e.g., Wiese et al., 2014). Therefore, although the learning paradigm used 501 here was designed in order to improve ecological validity in comparison with previous work, it 502 may be that further improvements could be made regarding the selection of the identities to be 503 learned.

504 In the current work, each participant was only tested once, with the delay interval varying 505 across the sample. A necessary limitation of this design was its inability to observe within506 participant memory decay and how this process may vary across individuals. An alternative 507 method of exploring the process of forgetting, therefore, would be to utilise a within-subjects 508 design, whereby each participant was tested at various intervals throughout the week. Although 509 certainly a more powerful approach statistically, the issue with this procedure is that participants 510 would be exposed to the faces during each test session. Such exposures would likely remind 511 participants of the faces to be remembered (even if different images were used) and would 512 therefore reinforce their memories artificially and improve accuracy on subsequent tests.

513 Related, it is widely known that testing itself aids learning (Larsen et al., 2009), even when no

514 feedback is given (Roediger \& Karpicke, 2006; for a review, see Roediger \& Butler, 2011). As a

515 result, simply testing participants throughout the week would artificially increase their 
516 performance and prevent the typical process of forgetting. In order to track forgetting over

517 multiple timepoints for a single individual, a different paradigm may be required.

518 In sum, this experiment adds to the sparse literature on the longitudinal process of

519 forgetting faces. Across seven days, I found that the majority of forgetting took place in the first

52024 hours, with no significant detriment after that period. In addition, self-reported face

521 recognition ability, and to a lesser extent personality, was predictive of task performance, and

522 these associations remained unchanged across delay intervals. Given that real-world forgetting

523 takes place over much longer time periods than typical studies consider, there is a growing need

524 for research investigating how face recognition deteriorates over the long term.

525

526 Acknowledgments

527 The author thanks Abi Davis for her critical comments throughout the project.

528

529 References

530 Andrews, S., Jenkins, R., Cursiter, H., \& Burton, A. M. (2015). Telling faces together: Learning 531 new faces through exposure to multiple instances. The Quarterly Journal of Experimental 532 Psychology, 68(10), 2041-2050.

533 Anwyl-Irvine, A. L., Massonnié, J., Flitton, A., Kirkham, N., \& Evershed, J. K. (2020). Gorilla

534 in our midst: An online behavioral experiment builder. Behavior Research Methods, 52(1), $535 \quad 388-407$.

536 Arizpe, J. M., Saad, E., Douglas, A. O., Germine, L., Wilmer, J. B., \& DeGutis, J. M. (2019).

537 Self-reported face recognition is highly valid, but alone is not highly discriminative of 
prosopagnosia-level performance on objective assessments. Behavior Research Methods, $51(3), 1102-1116$.

540 541

Averell, L., \& Heathcote, A. (2011). The form of the forgetting curve and the fate of memories. Journal of Mathematical Psychology, 55(1), 25-35.

Bahrick, H. P., Bahrick, P. O., \& Wittlinger, R. P. (1975). Fifty years of memory for names and faces: A cross-sectional approach. Journal of Experimental Psychology: General, 104(1), 54-75.

Baker, K. A., Laurence, S., \& Mondloch, C. J. (2017). How does a newly encountered face become familiar? The effect of within-person variability on adults' and children's perception of identity. Cognition, 161, 19-30.

Bobak, A. K., Mileva, V. R., \& Hancock, P. J. (2019). Facing the facts: Naive participants have only moderate insight into their face recognition and face perception abilities. Quarterly Journal of Experimental Psychology, 72(4), 872-881.

Bornstein, M. H., Hahn, C. S., \& Haynes, O. M. (2011). Maternal personality, parenting cognitions, and parenting practices. Developmental Psychology, 47(3), 658-675.

Bruce, V., Henderson, Z., Newman, C., \& Burton, A. M. (2001). Matching identities of familiar and unfamiliar faces caught on CCTV images. Journal of Experimental Psychology: Applied, 7(3), 207-218.

Burleigh, T., \& Leeper, T. J. (2020). pyMTurkR: A Client for the 'MTurk' Requester API. R package version 1.1.4. https://CRAN.R-project.org/package=pyMTurkR Burnham, M. J., Le, Y. K., \& Piedmont, R. L. (2018). Who is Mturk? Personal characteristics and sample consistency of these online workers. Mental Health, Religion \& Culture, 21(910), 934-944. 
561 Burton, A. M., Jenkins, R., Hancock, P. J., \& White, D. (2005). Robust representations for face

562

563

564

565

566

567

568

569

570

571

572

573

574

575

576

577 Courtois, M. R., \& Mueller, J. H. (1981). Target and distractor typicality in facial recognition? $578 \quad$ Journal of Applied Psychology, 66(5), 639-645.

579 Cunningham, J. A., Godinho, A., \& Kushnir, V. (2017). Using Mechanical Turk to recruit 580 participants for internet intervention research: Experience from recruitment for four trials 581 targeting hazardous alcohol consumption. BMC Medical Research Methodology, 17(1),

582 recognition: The power of averages. Cognitive Psychology, 51(3), 256-284.

Burton, A. M., Kramer, R. S. S., Ritchie, K. L., \& Jenkins, R. (2016). Identity from variation: Representations of faces derived from multiple instances. Cognitive Science, 40(1), 202223.

Burton, A. M., White, D., \& McNeill, A. (2010). The Glasgow Face Matching Test. Behavior Research Methods, 42(1), 286-291.

Burton, A. M., Wilson, S., Cowan, M., \& Bruce, V. (1999). Face recognition in poor-quality video: Evidence from security surveillance. Psychological Science, 10(3), 243-248.

Chandler, J., \& Shapiro, D. (2016). Conducting clinical research using crowdsourced convenience samples. Annual Review of Clinical Psychology, 12, 53-81.

Clutterbuck, R., \& Johnston, R. A. (2005). Demonstrating how unfamiliar faces become familiar using a face matching task. European Journal of Cognitive Psychology, 17(1), 97-116.

Costa, P.T., Jr., \& McCrae, R. R. (2008). The Revised NEO Personality Inventory (NEO-PI-R). In G. J. Boyle, G. Matthews, \& D. H. Saklofske (Eds.), The SAGE handbook of personality theory and assessment (2nd ed.) (pp. 179-198). SAGE. 156. 
583 Davis, J. P. (2019). The worldwide impact of identifying super-recognisers in police and 584 business. The Cognitive Psychology Bulletin, 4, 17-22.

585 Davis, J. P., Bretfelean, L. D., Belanova, E., \& Thompson, T. (2019). Assessing the long-term 586 face memory of highly superior and typical-ability short-term face recognisers. PsyArXiv. 587 https://doi.org/10.31234/osf.io/var4m

588 Davis, J. P., Bretfelean, L. D., Belanova, E., \& Thompson, T. (2020). Super-recognisers: Face 589 recognition performance after variable delay intervals. Applied Cognitive Psychology, $590 \quad 34(6), 1350-1368$.

591 Davis, J. P., Forrest, C., Treml, F., \& Jansari, A. (2018). Identification from CCTV: Assessing 592 police super-recogniser ability to spot faces in a crowd and susceptibility to change $593 \quad$ blindness. Applied Cognitive Psychology, 32(3), 337-353.

594 Davis, J. P., Lander, K., Evans, R., \& Jansari, A. (2016). Investigating predictors of superior face 595 recognition ability in police super-recognisers. Applied Cognitive Psychology, 30(6), 827$596 \quad 840$.

597 Davis, J. P., \& Tamonytė, D. (2017). Masters of disguise: Super-recognisers' superior memory 598 for concealed unfamiliar faces. Proceedings of the 2017 Seventh International Conference on Emerging Security Technologies (EST), 6-8 September 2017, Canterbury, UK.

600 Deffenbacher, K. A., Bornstein, B. H., McGorty, E. K., \& Penrod, S. D. (2008). Forgetting the 601 602 once-seen face: Estimating the strength of an eyewitness's memory representation. Journal 603 of Experimental Psychology: Applied, 14(2), 139-150.

Deffenbacher, K. A., Bornstein, B. H., Penrod, S. D., \& McGorty, E. K. (2004). A meta-analytic 604 605 review of the effects of high stress on eyewitness memory. Law and Human Behavior, $28(6), 687-706$. 
606 Devue, C., Wride, A., \& Grimshaw, G. M. (2019). New insights on real-world human face 607 recognition. Journal of Experimental Psychology: General, 148(6), 994-1007.

608 Diamond, R., \& Carey, S. (1986). Why faces are and are not special: An effect of expertise. 609 Journal of Experimental Psychology: General, 115(2), 107-117.

610 Duchaine, B., \& Nakayama, K. (2006). The Cambridge Face Memory Test: Results for

611

612

613

614

615

616

617

618

619

620

621

622

623

624

625

626

627 neurologically intact individuals and an investigation of its validity using inverted face stimuli and prosopagnosic participants. Neuropsychologia, 44(4), 576-585.

Ebbinghaus, H. (1885). Über das Gedächtnis [Memory]. Leipzig, Germany: Duncker and Humblot.

Ellis, H. D., Shepherd, J. W., \& Davies, G. M. (1979). Identification of familiar and unfamiliar faces from internal and external features: Some implications for theories of face recognition. Perception, 8(4), 431-439.

Ehrhart, M. G., Ehrhart, K. H., Roesch, S. C., Chung-Herrera, B. G., Nadler, K., \& Bradshaw, K. (2009). Testing the latent factor structure and construct validity of the Ten-Item Personality Inventory. Personality and Individual Differences, 47(8), 900-905.

Eysenck, H. J., \& Eysenck, S. B. G. (1993). Eysenck Personality Questionnaire-Revised. Hodder and Stoughton.

Faul, F., Erdfelder, E., Lang, A. -G., \& Buchner, A. (2007). G* Power 3: A flexible statistical power analysis program for the social, behavioral, and biomedical sciences. Behavior Research Methods, 39(2), 175-191.

Gosling, S. D., Rentfrow, P. J., \& Swann Jr, W. B. (2003). A very brief measure of the Big-Five personality domains. Journal of Research in Personality, 37(6), 504-528. 
628 Gray, K. L., Bird, G., \& Cook, R. (2017). Robust associations between the 20-item

629 prosopagnosia index and the Cambridge Face Memory Test in the general population.

$630 \quad$ Royal Society Open Science, 4(3), 160923.

631 Hancock, P. J. B., Bruce, V., \& Burton, A. M. (2000). Recognition of unfamiliar faces. Trends in 632 Cognitive Sciences, 4(9), 330-337.

633 Hauser, D. J., \& Schwarz, N. (2016). Attentive Turkers: MTurk participants perform better on 634 online attention checks than do subject pool participants. Behavior Research Methods, $635 \quad 48(1), 400-407$.

636 Holmes, M. (2010). A study to investigate the reliability and validity of the Ten-Item Personality 637 Inventory, when compared with two robust inventories, within a British sample. [B. Sc.

638 Thesis, York St John University]. Retrieved from http://e-

639 space.mmu.ac.uk/576699/1/Holmes\%20York\%20St\%20John.pdf

640 Jenkins, R., White, D., Van Montfort, X., \& Burton, A. M. (2011). Variability in photos of the 641 same face. Cognition, 121, 313-323.

642 Kennerknecht, I., Ho, N. Y., \& Wong, V. C. (2008). Prevalence of hereditary prosopagnosia

643

644

645

646

647

648

649 (HPA) in Hong Kong Chinese population. American Journal of Medical Genetics Part A, $146(22), 2863-2870$.

Kramer, R. S. S., Hardy, S. C., \& Ritchie, K. L. (2020). Searching for faces in crowd chokepoint videos. Applied Cognitive Psychology, 34(2), 343-356.

Kramer, R. S. S., Manesi, Z., Towler, A., Reynolds, M. G., \& Burton, A. M. (2018). Familiarity and within-person facial variability: The importance of the internal and external features. Perception, 47(1), 3-15. 
650 Krzanowski, W. J., \& Hand, D. J. (2009). ROC curves for continuous data. London: Chapman \& $651 \quad$ Hall.

652 Lander, K., \& Bruce, V. (2003). The role of motion in learning new faces. Visual Cognition, $653 \quad 10(8), 897-912$.

654 Lander, K., \& Davies, R. (2007). Exploring the role of characteristic motion when learning new 655 faces. Quarterly Journal of Experimental Psychology, 60(4), 519-526.

656 Lander, K., \& Poyarekar, S. (2015). Famous face recognition, face matching, and extraversion. 657 Quarterly Journal of Experimental Psychology, 68(9), 1769-1776.

658 Larsen, D. P., Butler, A. C., \& Roediger, H. L., III. (2009). Repeated testing improves long-term 659 retention relative to repeated study: A randomised controlled trial. Medical Education, $660 \quad 43(12), 1174-1181$.

661 Li, J., Tian, M., Fang, H., Xu, M., Li, H., \& Liu, J. (2010). Extraversion predicts individual 662 differences in face recognition. Communicative \& Integrative Biology, 3(4), 295-298.

663 Livingston, L. A., \& Shah, P. (2018). People with and without prosopagnosia have insight into 664 their face recognition ability. Quarterly Journal of Experimental Psychology, 71(5), 1260$665 \quad 1262$.

666 Matsuyoshi, D., \& Watanabe, K. (2020). People have modest, not good, insight into their face 667 recognition ability: A comparison between self-report questionnaires. Psychological 668 Research. Advance online publication.

669 McCaffery, J. M., Robertson, D. J., Young, A. W., \& Burton, A. M. (2018). Individual 670 differences in face identity processing. Cognitive Research: Principles and Implications, 3, 67121. 
672 Megreya, A. M., \& Bindemann, M. (2013). Individual differences in personality and face 673 identification. Journal of Cognitive Psychology, 25(1), 30-37.

674 Murre, J. M., \& Chessa, A. G. (2011). Power laws from individual differences in learning and 675 forgetting: Mathematical analyses. Psychonomic Bulletin \& Review, 18(3), 592-597.

676 Palermo, R., Rossion, B., Rhodes, G., Laguesse, R., Tez, T., Hall, B., Albonico, A., Malaspina, 677 M., Daini, R., Irons, J., Al-Janabi, S., Taylor, L. C., Rivolta, D., \& McKone, E. (2017). Do 678 people have insight into their face recognition abilities? Quarterly Journal of Experimental 679 Psychology, 70(2), 218-233.

680 Ritchie, K. L., \& Burton, A. M. (2017). Learning faces from variability. Quarterly Journal of 681 Experimental Psychology, 70(5), 897-905.

682 Ritchie, K. L., Smith, F. G., Jenkins, R., Bindemann, M., White, D., \& Burton, A. M. (2015).

683 Viewers base estimates of face matching accuracy on their own familiarity: Explaining the 684 photo-ID paradox. Cognition, 141, 161-169.

685 Roberts, B. W., Walton, K. E., \& Viechtbauer, W. (2006). Patterns of mean-level change in 686 personality traits across the life course: A meta-analysis of longitudinal studies.

687 Psychological Bulletin, 132, 3-27.

688 Roediger, H. L., III., \& Butler, A. C. (2011). The critical role of retrieval practice in long-term 689 retention. Trends in Cognitive Sciences, 15(1), 20-27.

690 Roediger, H. L., III., \& Karpicke, J. D. (2006). Test-enhanced learning: Taking memory tests 691 improves long-term retention. Psychological Science, 17(3), 249-255.

692 Rubin, D. C., \& Wenzel, A. E. (1996). One hundred years of forgetting: A quantitative 693 description of retention. Psychological Review, 103(4), 734-760. 
694 Rule, N. O., Slepian, M. L., \& Ambady, N. (2012). A memory advantage for untrustworthy 695 faces. Cognition, 125(2), 207-218.

696 Russell, R., Duchaine, B., \& Nakayama, K. (2009). Super-recognizers: People with extraordinary 697 face recognition ability. Psychonomic Bulletin \& Review, 16(2), 252-257.

698 Saraiva, R. B., van Boeijen, I. M., Hope, L., Horselenberg, R., Sauerland, M., \& van Koppen, P. 699 J. (2019). Development and validation of the Eyewitness Metamemory Scale. Applied

700 Cognitive Psychology, 33(5), 964-973.

701

702

703

704

705

706

707

708

709

710

711

712

713

714

715

Sauer, J., Brewer, N., Zweck, T., \& Weber, N. (2010). The effect of retention interval on the confidence-accuracy relationship for eyewitness identification. Law and Human Behavior, $34(4), 337-347$.

Shah, P., Gaule, A., Sowden, S., Bird, G., \& Cook, R. (2015). The 20-item prosopagnosia index (PI20): A self-report instrument for identifying developmental prosopagnosia. Royal Society Open Science, 2(6), 140343.

Shah, P., Sowden, S., Gaule, A., Catmur, C., \& Bird, G. (2015). The 20 item prosopagnosia index (PI20): Relationship with the Glasgow face-matching test. Royal Society Open Science, 2(11), 150305.

Shakeshaft, N. G., \& Plomin, R. (2015). Genetic specificity of face recognition. Proceedings of the National Academy of Sciences, 112(41), 12887-12892.

Shapiro, D. N., Chandler, J., \& Mueller, P. A. (2013). Using Mechanical Turk to study clinical populations. Clinical Psychological Science, 1(2), 213-220.

Shapiro, P. N., \& Penrod, S. (1986). Meta-analysis of facial identification studies. Psychological Bulletin, 100, 139-156. 
716 Shepherd, J. W., \& Ellis, H. D. (1973). The effect of attractiveness on recognition memory for 717 faces. American Journal of Psychology, 86, 627-633.

718 Shepherd, J. W., Ellis, H. D., \& Davies, G. M. (1982). Identification evidence: A psychological 719 evaluation. Aberdeen, Scotland: Aberdeen University Press.

720 Shepherd, J. W., Gibling, F., \& Ellis, H. D. (1991). The effects of distinctiveness, presentation 721 time and delay on face recognition. European Journal of Cognitive Psychology, 3(1), 137722 145.

723

724

725

726

727

728

729

730

731

732

733

734

735

736

737

738

Sheth, B. R., Nguyen, N., \& Janvelyan, D. (2009). Does sleep really influence face recognition memory? PLoS ONE, 4(5), e5496.

Stephan, Y., Sutin, A. R., Luchetti, M., \& Terracciano, A. (2020). Personality and memory performance over twenty years: Findings from three prospective studies. Journal of Psychosomatic Research, 128, 109885.

Stoycheff, E. (2016). Please participate in Part 2: Maximizing response rates in longitudinal MTurk designs. Methodological Innovations, 9, 1-5.

Ventura, P., Livingston, L. A., \& Shah, P. (2018). Adults have moderate-to-good insight into their face recognition ability: Further validation of the 20-item Prosopagnosia Index in a Portuguese sample. Quarterly Journal of Experimental Psychology, 71(12), 2677-2679.

Wagner, U., Kashyap, N., Diekelmann, S., \& Born, J. (2007). The impact of post-learning sleep vs. wakefulness on recognition memory for faces with different facial expressions. Neurobiology of Learning and Memory, 87(4), 679-687.

Wiese, H., Altmann, C. S., \& Schweinberger, S. R. (2014). Effects of attractiveness on face memory separated from distinctiveness: Evidence from event-related brain potentials. Neuropsychologia, 56, 26-36. 
739 Wilmer, J. B., Germine, L., Chabris, C. F., Chatterjee, G., Williams, M., Loken, E., Nakayama,

740 K., \& Duchaine, B. (2010). Human face recognition ability is specific and highly heritable.

$741 \quad$ Proceedings of the National Academy of Sciences of the United States of America, 107(11),

$742 \quad 5238-5241$.

743 Wixted, J. T., \& Ebbesen, E. B. (1991). On the form of forgetting. Psychological Science, 2(6), $744 \quad 409-415$.

745 Yarmey, A. D. (1979). The effects of attractiveness, feature saliency and liking on memory for 746 faces. In M. Cook \& G. Wilson (Eds.), Love and attraction (pp. 51-53). Oxford, England: 747 Pergamon Press.

748 Young, A. W., \& Burton, A. M. (2018). Are we face experts? Trends in Cognitive Sciences, $749 \quad 22(2), 100-110$.

750 Zhou, X., Matthews, C. M., Baker, K. A., \& Mondloch, C. J. (2018). Becoming familiar with a 751 newly encountered face: Evidence of an own-race advantage. Perception, 47(8), 807-820.

752

753 


\section{Figure Captions}

755

756 Figure 1. Images of the same identity, representative of the videos presented during the learning 757 task (left) and images presented during the recognition test (right). Photo credits: Robin Kramer. 758

759 Figure 2. The effect of delay on face recognition performance. The dashed line depicts a power 760 model for this relationship. Error bars represent 95\% confidence intervals. 


\section{Table 1 (on next page)}

Summary of sample size and exclusion information for each condition. 
1 Table 1. Summary of sample size and exclusion information for each condition.

\begin{tabular}{|c|c|c|c|c|c|c|}
\hline & \multicolumn{5}{|c|}{ Delay } \\
\hline & & None & 6 hours & 12 hours & 1 day & 7 days \\
\hline \multirow[t]{4}{*}{ Learning } & Completed & 235 & 478 & 571 & 400 & 401 \\
\hline & Excluded - attention checks & 63 & 140 & 140 & 144 & 130 \\
\hline & Excluded - familiarity check & - & 59 & 69 & 53 & 46 \\
\hline & Final sample & - & 279 & 362 & 203 & 225 \\
\hline \multirow[t]{4}{*}{ Testing } & Completed & - & 119 & 110 & 161 & 165 \\
\hline & Excluded - attention checks & 27 & 6 & 5 & 19 & 14 \\
\hline & Excluded - familiarity check & 32 & 11 & 5 & 16 & 22 \\
\hline & Final sample & 113 & 102 & 100 & 126 & 129 \\
\hline
\end{tabular}

2 
Table 2 (on next page)

Summary data for participants' responses. 
1 Table 2. Summary data for participants' responses.

\begin{tabular}{|c|c|c|c|c|c|c|c|c|}
\hline Condition & Delay (hours) & AUC & HK11 & $E$ & $\mathrm{~A}$ & $\mathrm{C}$ & ES & $\mathrm{O}$ \\
\hline 12 hours & $12.28(0.54)$ & $0.73(0.12)$ & $22.31(6.48)$ & $3.47(1.56)$ & $5.03(1.40)$ & $5.73(1.13)$ & $4.97(1.42)$ & $4.87(1.25)$ \\
\hline 7 days & $173.26(5.68)$ & $0.60(0.14)$ & $23.70(7.40)$ & $3.72(1.62)$ & $5.09(1.25)$ & $5.47(1.33)$ & $4.93(1.50)$ & $4.89(1.26)$ \\
\hline All participants & $49.55(68.02)$ & $0.67(0.15)$ & $23.56(7.30)$ & $3.68(1.55)$ & $5.02(1.27)$ & $5.49(1.29)$ & $4.85(1.42)$ & $4.92(1.28)$ \\
\hline
\end{tabular}

$2 \quad$ Note. $\mathrm{E}=$ Extraversion; $\mathrm{A}=$ Agreeableness; $\mathrm{C}=$ Conscientiousness; $\mathrm{ES}=$ Emotional Stability; $\mathrm{O}=$ Openness. Values are presented as 3 $M(S D)$. 


\section{Table 3 (on next page)}

The hierarchical regression analysis for predicting performance (AUC). 
1 Table 3. The hierarchical regression analysis for predicting performance (AUC).

\begin{tabular}{|c|c|c|c|c|c|c|c|}
\hline & Variable & $B$ & $S E$ & $\beta$ & $t$ & $R^{2}$ & $\Delta R^{2}$ \\
\hline \multirow[t]{6}{*}{ Step 1} & & & & & & 0.14 & 0.14 \\
\hline & Intercept & 0.75 & 0.01 & & $55.33 * * *$ & & \\
\hline & Delay: 6 hours & -0.05 & 0.02 & -0.13 & $-2.63 * *$ & & \\
\hline & Delay: 12 hours & -0.02 & 0.02 & -0.05 & -1.12 & & \\
\hline & Delay: 1 day & -0.13 & 0.02 & -0.34 & $-6.85 * * *$ & & \\
\hline & Delay: 7 days & -0.14 & 0.02 & -0.39 & $-7.81 * * *$ & & \\
\hline \multirow[t]{7}{*}{ Step 2} & & & & & & 0.25 & 0.11 \\
\hline & Intercept & 0.92 & 0.02 & & $40.41 * * *$ & & \\
\hline & Delay: 6 hours & -0.06 & 0.02 & -0.16 & $-3.52 * * *$ & & \\
\hline & Delay: 12 hours & -0.04 & 0.02 & -0.09 & $-2.00 *$ & & \\
\hline & Delay: 1 day & -0.13 & 0.02 & -0.34 & $-7.33 * * *$ & & \\
\hline & Delay: 7 days & -0.15 & 0.02 & -0.41 & $-8.66 * * *$ & & \\
\hline & HK11 & -0.01 & 0.00 & -0.33 & $-9.12 * * *$ & & \\
\hline \multirow[t]{8}{*}{ Step 3} & & & & & & 0.27 & 0.02 \\
\hline & Intercept & 0.98 & 0.03 & & $35.78 * * *$ & & \\
\hline & Delay: 6 hours & -0.07 & 0.02 & -0.17 & $-3.80 * * *$ & & \\
\hline & Delay: 12 hours & -0.04 & 0.02 & -0.10 & $-2.29 *$ & & \\
\hline & Delay: 1 day & -0.13 & 0.02 & -0.34 & $-7.34 * * *$ & & \\
\hline & Delay: 7 days & -0.15 & 0.02 & -0.41 & $-8.82 * * *$ & & \\
\hline & HK11 & -0.01 & 0.00 & -0.35 & $-9.61 * * *$ & & \\
\hline & Extraversion & -0.01 & 0.00 & -0.14 & $-3.90 * * *$ & & \\
\hline
\end{tabular}


2 Note. Delay reference category $=$ no delay. ${ }^{*} p<.05, * * p<.01, * * * p<.001$ 


\section{Figure 1}

Images of the same identity, representative of the videos presented during the learning task (left) and images presented during the recognition test (right).

Photo credits: Robin Kramer.

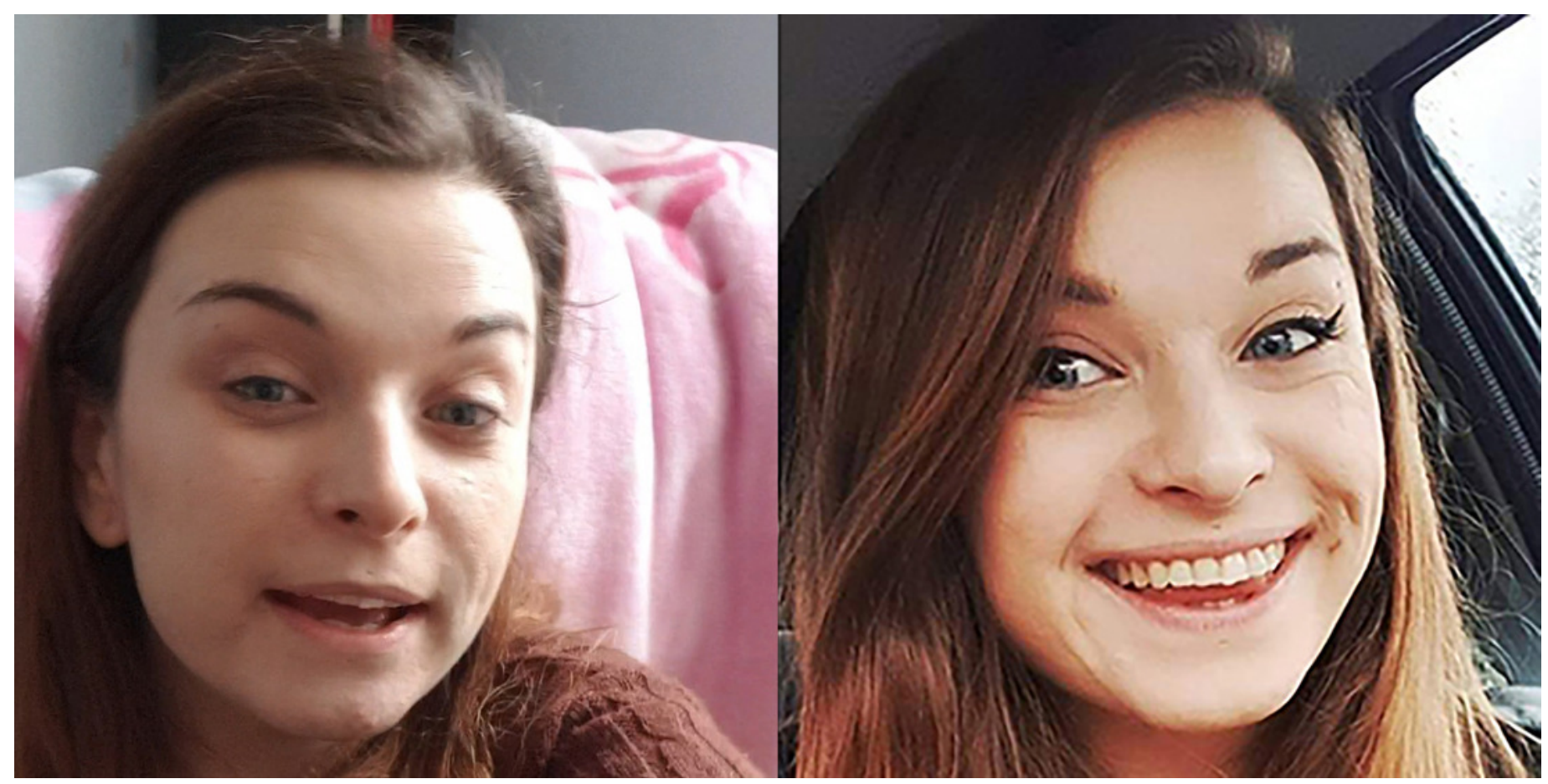


Figure 2

The effect of delay on face recognition performance.

The dashed line depicts a power model for this relationship. Error bars represent 95\% confidence intervals.

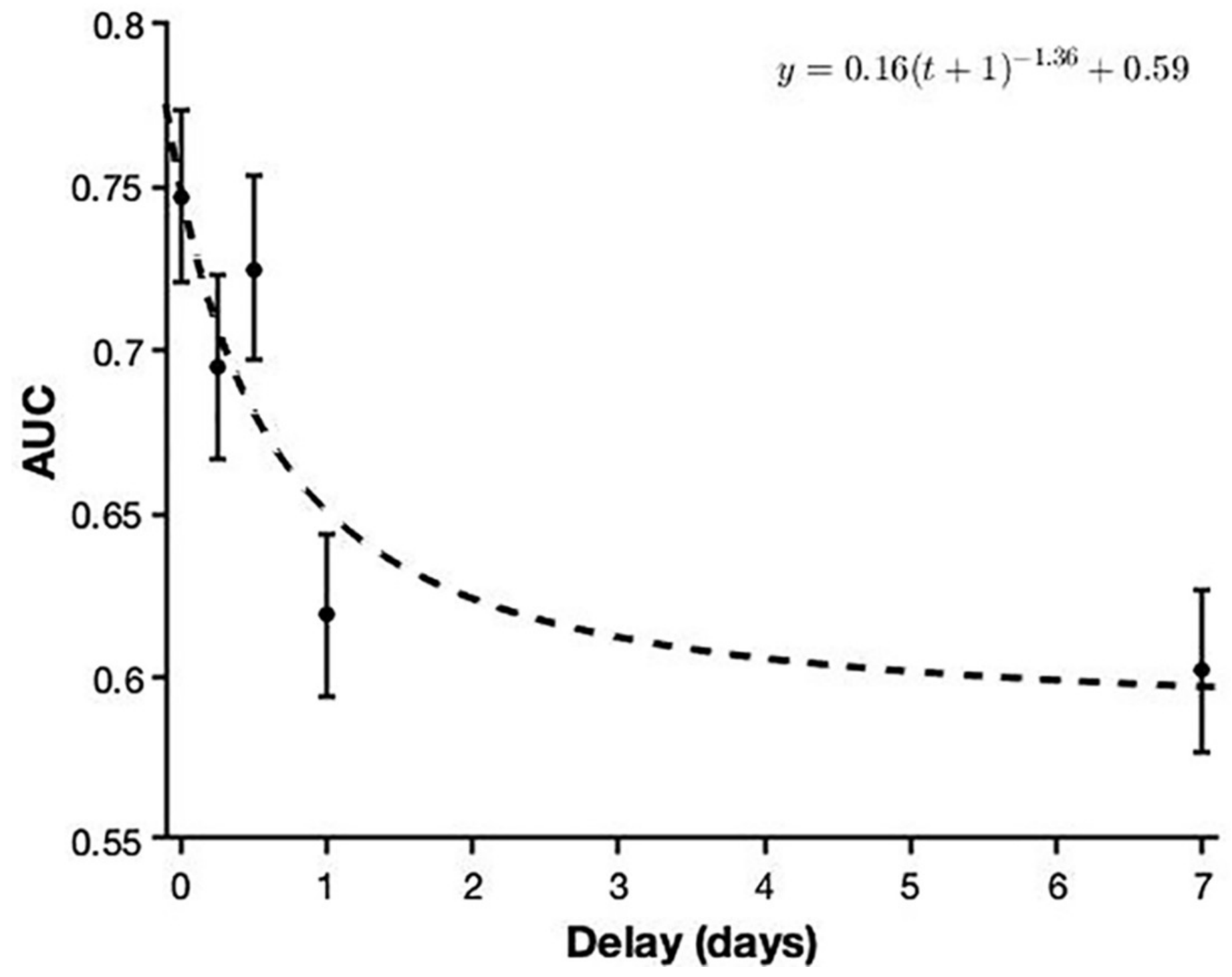

